An official journal of the / Un journal officiel de la "Société Sénégalaise de Cancérologie" (SOSECAN)

Journal homepage: www.africanjournalofoncology.com

\title{
Giant jejunal gasto-intestinal stromal tumor: A rare cause of abdominal mass in an elderly female
}

Tumeur stromale gastro-intestinale à localisation jéjunale : Une rare cause de masse abdominale chez la femme âgée

\author{
AC. Diallo', A. Ndong', I. Niang', MB. Ba ${ }^{3}$, JA. Thiam³, PS. Dieng' ${ }^{3}$ S. Ka ${ }^{3}$ I. Konate', A. Dem³
}

' UFR 2S, Université Gaston Berger de Saint-Louis, Sénégal.

${ }^{2}$ Service de Radiologie, Centre Hospitalier de Fann, Sénégal.

${ }^{3}$ Institut Joliot Curie de Dakar, Sénégal.

OBJECTIVE: We report the case of a patient presenting an abdominal mass whose final diagnosis is a gastrointestinal stromal tumor (GIST). CLINICAL OBSERVATION: It was a 61 -year-old patient with no pathological history received for a progressive increase in the volume of the abdomen evolving for one year and painless. On examination, the patient was in good general condition, with normal patterns. There was an abdomino-pelvic mass of about $20 \mathrm{~cm}$ long axis, mobile. The rest of the exam was unremarkable. The biological assessment was normal. Abdominopelvic computed tomography revealed a tissue mass with necrosis. During the surgical exploration, a mass developed on the mesenteric border $45 \mathrm{~cm}$ from the Treitz angle was noted. It is not associated with ascites or peritoneal carcinoma. Bowel resection removing the mass was performed followed by end-to-end anastomosis. Pathological examination of the surgical specimen diagnosed GIST. The postoperative course at three months was excellent both clinically and radiologically. CONCLUSION: The case that we report underlines the possible jejunal localization of GIST and the clinical presentation may be usual. It also shows the difficulty of obtaining a preoperative diagnosis and the central role of surgery in management.

KEYWORDS: Gastrointestinal stromal tumor; Jejunal GIST; Surgery; Bowel resection; Imatinib.

OBJECTIF : Nous rapportons le cas d'une patiente présentant une masse abdominale dont le diagnostic final est une tumeur stromale gastro-intestinale (GIST). OBSERVATION : II s'agissait d'une patiente âgée de $6 \mathrm{I}$ ans sans antécédents pathologiques, reçue pour une augmentation progressive de volume de l'abdomen évoluant depuis un an, indolore. A l'examen, la patiente avait un bon état général, des constantes normales. Nous notions une masse abdomino-pelvienne d'environ $20 \mathrm{~cm}$ de grand axe, mobile. Le reste de l'examen était sans particularité. Le bilan biologique était normal. La tomodensitométrie abdominopelvienne mettait en évidence une masse tissulaire avec des remaniements nécrotiques. Une indication opératoire a été posée. A l'exploration, nous notions une masse développée sur le bord mésentérique à $45 \mathrm{~cm}$ de l'angle de Treitz. Elle n'est pas associée à de l'ascite ou à une carcinose péritonéale. II n'y avait pas de lésion hépatique également. La résection intestinale emportant la masse a été réalisée suivie d'une anastomose termino-terminale. Les suites opératoires étaient simples. L'examen anatomo-pathologique de la pièce opératoire a posé le diagnostic d'une GIST. L'évolution post-opératoire à trois mois était excellente tant sur le plan clinique que radiologique. CONCLUSION : Le cas que nous rapportons souligne la possibilité de retrouver une GIST à localisation jéjunale malgré une présentation clinique peu usuelle. Elle montre également la difficulté d'obtenir un diagnostic préopératoire et le rôle central de la chirurgie dans la prise en charge.

MOTS-CLES : Tumeur stromale gastro-intestinale ; GIST jéjunale ; Chirurgie ; Résection intestinale ; Imatinib.

\section{INTRODUCTION}

Les tumeurs stromales gastro-intestinales (GIST) sont des tumeurs mésenchymateuses rares [I]. La localisation gastrique est la plus fréquente (60\%) [2]. L'atteinte jéjunale est beaucoup plus rare ( 3 à $5 \%$ ). La symptomatologie varie selon le siège et l'évolution. Elle est souvent peu spécifique. Dans environ la moitié des cas, les patients présentent une masse abdominale et dans moins de $1 \%$ des cas, une hémorragie digestive [3]. Nous rapportons le cas d'une patiente avec une GIST à localisation jéjunale avec une symptomatologie peu fréquente.

\section{OBSERVATION CLINIQUE}

II s'agissait d'une patiente âgée de $6 \mathrm{I}$ ans sans antécédents pathologiques, reçue pour une augmentation progressive de volume de l'abdomen évoluant depuis un ans, indolore. Elle présentait plusieurs épisodes de mélaena. A l'examen, la patiente avait un bon état général, des constantes normales. Nous notions une masse abdomino-pelvienne d'environ $20 \mathrm{~cm}$ de grand axe, mobile. Le reste de l'examen était sans particularité. Le bilan biologique était normal. La tomodensitométrie abdominopelvienne (TDM) mettait en évidence une masse volumineuse masse tissulaire de $18 \times 12 \times$ $18 \mathrm{~cm}$, avec des remaniements nécrotiques sans possibilité d'être formelle sur son origine. II n'y avait pas d'autre lésion abdominopelvienne visualisée (Figure I). Une indication opératoire a été posée. A l'exploration, nous notions une masse développée sur le bord mésentérique à $45 \mathrm{~cm}$ de l'angle de Treitz (Figure 2). Elle n'est pas associée à de l'ascite ou à 
une carcinose péritonéale. II n’y avait pas de lésion hépatique également.

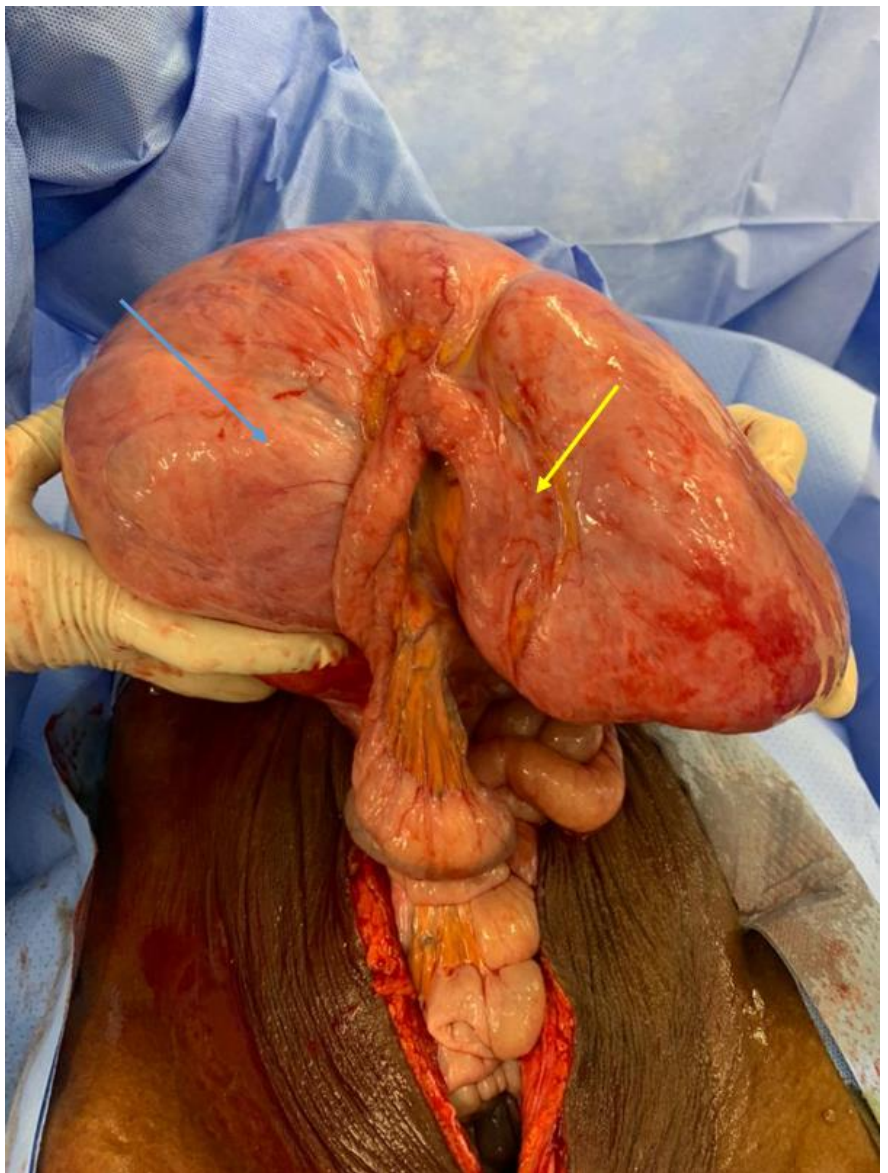

Figure I : Aspect peropératoire de la masse (flèche bleue) développée aux dépens de la première anse jéjunale sur son bord mésentérique (flèche jaune).

La résection intestinale emportant la masse a été réalisée suivie d'une anastomose termino-terminale. Les suites opératoires étaient simples. L'examen anatomo-pathologique de la pièce opératoire avait objectivé des cellules fusiformes grêliques infiltrant la sous séreuse. L'étude immunohistochimique montrait un marquage net diffus de la population tumorale visà-vis du Ckit et du DOGI, le marquage de l'actine muscle lisse et de la PSI00 étaient négatif. L'aspect morphologique et immunohistochimique correspondait donc à une GIST à localisation jéjunale et à indice mitotique faible (inférieur à cinq). L'évolution post-opératoire à trois mois était excellente tant sur le plan clinique que radiologique.

\section{DISCUSSION}

Les GIST sont des tumeurs rares et leur diagnostic est rarement aisé. Elles sont souvent asymptomatiques ou donnent des symptômes peu spécifiques [4, 5]. Les GIST surviennent surtout chez les adultes avec une médiane d'âge de 65 ans [4]. II n'existe pas de prédominance particulière en fonction du sexe dans la plupart des séries publiées [5]. Les circonstances de découverte sont multiples et non spécifiques. La localisation gastrique est plus fréquemment retrouvée comparée à la localisation jéjunale [6, 7]. Le plus souvent, les GIST à localisation jéjunale sont asymptomatiques. Elles se présentent le plus souvent sous la forme d'une masse avec une taille moyenne plus importante. Le diagnostic peut être posé rarement à la suite d'une hémorragie digestive [4, 6]. En effet, les GIST sont responsables dans 5 à $10 \%$ des causes de saignements digestifs occultes [4]. Dans notre cas, la patiente avait des épisodes de mélaena.

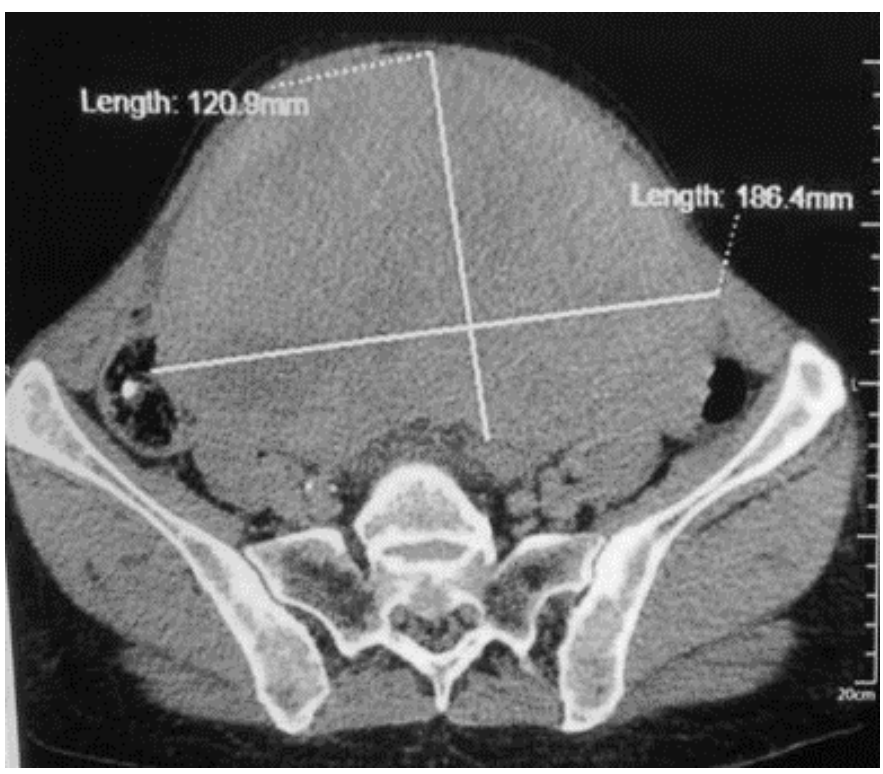

Figure 2A.

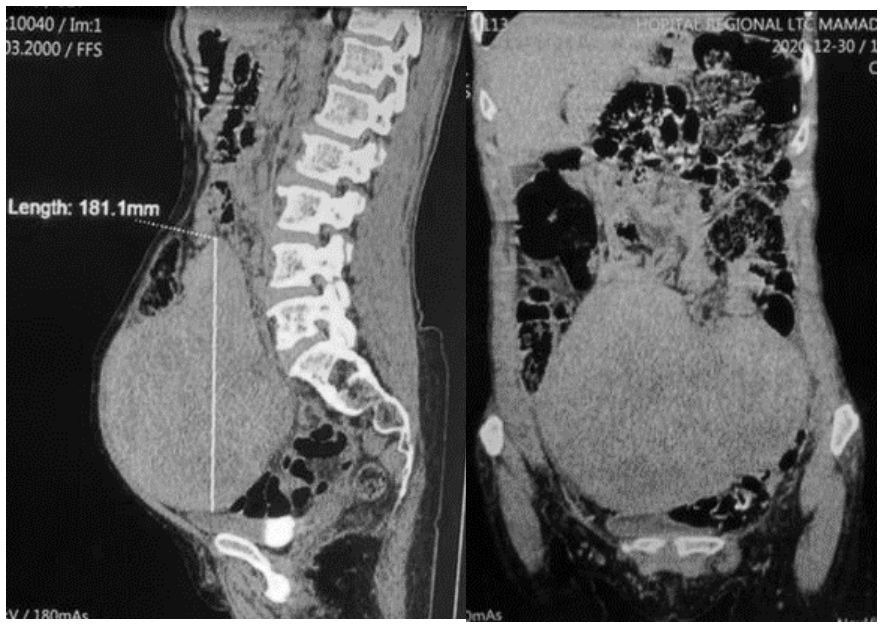

Figure 2B.

Figure 2 : TDM en coupe transversale avec reconstruction coronale (2A) et sagittale (2B) montrant la volumineuse masse tissulaire abdominopelvienne.

Le plus souvent, la tumeur se développe de façon extraluminale rendant l'endoscopie digestive et la biopsie peu contributive $[4,8]$. La TDM occupe une place centrale dans le cadre du diagnostic des GIST à localisation grêlique [9]. Elle permet de caractériser la tumeur, sa taille et son extension locorégionale.

Le diagnostic histologique est rarement obtenu en préopératoire. En effet, la biopsie entraine un haut risque de dissémination ou d'hémorragie. Il est surtout nécessaire en cas de tumeur métastatique ou non-résécable. L'aspect histologique des GIST est variable. Les cellules peuvent être fusiformes dans $70 \%$ des cas, épithélioïdes dans $20 \%$ des cas ou une combinaison de ces deux aspects $[10,1 \mathrm{I}]$. Le diagnostic anatomopathologique repose sur l'histopathologie et l'immunohistochimie. La recherche d'une expression des antigènes CDI I7, positive dans $95 \%$ des cas, permet d'affirmer le diagnostic. Cependant, il faut noter l'existence de faux positifs [12]. 
La chirurgie malgré l'apport de l'imatinib est le principal traitement curatif des GIST. II est recommandé de réaliser une résection complète avec des marges saines [7]. Les marges de résection doivent être au moins deux $\mathrm{cm}$ [13]. Du fait de l'absence d'atteinte ganglionnaire dans la grande majorité des cas, le curage ganglionnaire n'est pas systématique [ $\mathrm{I} \mathrm{I}$ ].

Les facteurs pronostiques sont la taille tumorale supérieure à $10 \mathrm{~cm}$, le siège de la tumeur, l'atteinte de la séreuse, un index mitotique (supérieur à 5 mitoses par 50 champs au fort grossissement), l'existence d'atypies nucléaires, l'état des marges de résection et l'existence d'une mutation Ckit [14]. L'exérèse chirurgicale complète assure $95 \%$ de survie après cinq ans.

\section{CONCLUSION}

Le cas que nous rapportons souligne la possible localisation jéjunale des GIST et la présentation clinique peu usuelle. II montre également la difficulté d'obtenir un diagnostic préopératoire et le rôle central de la chirurgie dans la prise en charge.

\section{CONFLITS D'INTERET}

Les auteurs n'ont déclaré aucun conflit d'intérêts.

\section{REFERENCES}

I. Global epidemiology of gastrointestinal stromal tumours (GIST): A systematic review of populationbased cohort studies. Cancer Epidemiol. 2016 Feb I;40:39-46.

2. Keung EZ, Raut CP. Management of Gastrointestinal Stromal Tumors. Surg Clin North Am. 2017 Apr I;97(2):437-52.

3. Roy SD, Khan D, De KK, De U. Spontaneous perforation of jejunal gastrintestinal stromal tumour (gist). Case report and review of literature. World J Emerg Surg. 2012 Nov 29;7(I):37.

4. Melo C, Canhoto C, Manata F, Bernardes A. Surgical treatment of giant gist with acute gastrointestinal bleeding: Case report. Int J Surg Case Rep. 2018 Jan I;53:354-7.

5. Somé OR, Tankoano IA, Koura M, Konségré $V$, Konkobo D, Koulidiaty J. Hepatic Metastatic GIST: Diagnostic and Therapeutic Difficulties in Souro Sanou Teaching Hospital in Bobo Dioulasso, Burkina Faso. Open J Gastroenterol. 2018 Dec 27;08(I 2):455.

6. Fall $F$, Ndiaye $B, N$ diaye $A R$, Diagne Guèye NM, Gning $\mathrm{SB}$, Niang $A$, et al. Les tumeurs stromales digestives " GIST » : étude rétrospective de dix cas au Sénégal. J Afr Hépato-Gastroentérologie. 201 I Jul 30;5(3):210.

7. Ka S, T M, Diallo A, T J, D A. Voluminous Gastric Stromal Tumors: Place of Surgery about 5 Cases at the Dakar Cancer Institute. J Gastrointest Cancer Stromal Tumors. 2017 Jan 1;02.

8. Akahoshi K, Oya M, Koga T, Shiratsuchi Y. Current clinical management of gastrointestinal stromal tumor. World J Gastroenterol. 2018 Jul 14;24(26):2806-17.

9. Burkill GJC, Badran M, Al-Muderis O, Meirion Thomas J, Judson IR, Fisher C, et al. Malignant Gastrointestinal Stromal Tumor: Distribution, Imaging Features, and Pattern of Metastatic Spread. Radiology. 2003 Feb;226(2):527-32.
10. Demetri GD, Benjamin RS, Blanke CD, Blay J-Y, Casali $P$, Choi $H$, et al. NCCN Task Force Report: Management of Patients with Gastrointestinal Stromal Tumor (GIST)-Update of the NCCN Clinical Practice Guidelines. J Natl Compr Canc Netw. 2007 May I;5(S2):S-S-29.

II. Zwan SM van der, DeMatteo RP. Gastrointestinal stromal tumor: 5 years later. Cancer. 2005; | 04(9): | 78|-8.

12. Dow N, Giblen G, Sobin LH, Miettinen M. Gastrointestinal stromal tumors: Differential diagnosis. Semin Diagn Pathol. 2006 May I;23(2): I I I9.

13. Barbu LA, Margaritescu N-D, Ghilusi M-C, Belivaca D, Georgescu EF, Ghelase M, et al. Severe upper gastrointestinal bleeding from gastrointestinal stromal tumor of the stomach. Rom J Morphol Embryol. 2016;57(4): I397-40।.

14. Caterino S, Lorenzon L, Petrucciani N, lannicelli E, Pilozzi E, Romiti A, et al. Gastrointestinal stromal tumors: correlation between symptoms at presentation, tumor location and prognostic factors in 47 consecutive patients. World J Surg Oncol. 201 I Feb $1 ; 9: 13$. 